\title{
BMJ Open Protocol of a scoping review assessing injury rates and their determinants among healthcare workers in western countries
}

\author{
Nicola Luigi Bragazzi, ${ }^{1}$ Guglielmo Dini, ${ }^{1,2}$ Valentina Parodi, ${ }^{1}$ Carlo Blasi, ${ }^{3}$ \\ Roberta Linares, ${ }^{3}$ Virginia Mortara, ${ }^{3}$ Alessandra Toletone, ${ }^{1,4}$ Francesca Maria Bersi, ${ }^{1}$ \\ Beatrice D'Amico, ${ }^{1,2}$ Emanuela Massa, ${ }^{1}$ Alfredo Montecucco, ${ }^{1,2}$ \\ Nicoletta Debarbieri, ${ }^{2}$ Paolo Durando ${ }^{1,2}$
}

To cite: Bragazzi NL, Dini G, Parodi V, et al. Protocol of a scoping review assessing injury rates and their determinants among healthcare workers in western countries. BMJ Open 2019;9:e023372. doi:10.1136/ bmjopen-2018-023372

- Prepublication history for this paper is available online To view these files, please visit the journal online (http://dx.doi org/10.1136/bmjopen-2018023372).

NLB and GD contributed equally.

Received 4 April 2018

Revised 30 November 2018

Accepted 4 December 2018

\section{ABSTRACT}

Introduction Healthcare workers (HCWs) are exposed to various risk factors and risky behaviours that may seriously affect their health and ability to work. The aim of this protocol is to detail the steps to follow in order to carry out a scoping review to assess the prevalence/incidence of injuries among HCWs.

Methods and analysis The study will be carried out in accordance with the Preferred Reporting Items for Systematic Reviews and Meta-Analyses-Protocols guidelines. Studies will be selected according to the following criteria: $\mathrm{P}$ (HCWs), $\mathrm{E}$ (exposure to injuries), $\mathrm{C}$ (different types of exposure and different categories of HCWs) and 0 (prevalence/incidence and determinants of injuries). A time filter has been set (literature between 2000 and 2018) to enable updated, direct comparison between the findings and the epidemiological data available at national and local 'Istituto Nazionale per I'Assicurazione contro gli Infortuni sul Lavoro' (National Institute for Insurance Against Accidents at Work) centres in Italy. No language restriction will be applied.

Ethics and dissemination Formal ethical approval is not required; primary data will not be collected, as they have already been published. The results will be disseminated through peer-reviewed publication(s), conference presentation(s) and the press.

\section{BACKGROUND}

The healthcare system is the fastest-growing sector in western countries and employs millions of workers: over 18 million in the USA and more than 59 million worldwide. 'Healthcare workers' (HCWs) is an umbrella term which refers to all people engaged in the promotion, protection, care or improvement of the health of the population. This term includes a variety of different figures, ranging from medical doctors (such as specialists, paediatricians and general practitioners) to midwives and nurses, other allied health professionals, central supply workers and technicians, and residents. ${ }^{2-4}$

\section{Strengths and limitations of this study}

- Current literature reports no reviews of studies performed in western countries concerning injuries among healthcare workers.

- Stratifying injuries according to type and the work tasks involved could add meaningful information.

- A major limitation concerns the time filter (studies published between 2000 and 2018). However, this enables updated, direct comparison between the findings and the epidemiological data available at national and local 'Istituto Nazionale per I'Assicurazione contro gli Infortuni sul Lavoro' (National Institute for Insurance Against Accidents at Work) centres.

Several variables may constitute risk factors and impact on HCWs' health and safety, potentially leading to occupational injuries and diseases. These include: the individual characteristics of the HCWs (age, gender, education, smoking status and other lifestyle habits) and the features of the patient under care (sociodemographic characteristics and type of disease) and of the healthcare setting (eg, organisation, workload or night shifts) and procedures (such as invasive treatment).

Like other working environments, hospitals and other healthcare facilities are not completely safe workplaces for professionals. HCWs may be exposed to several occupational health hazards which may impose a considerable clinical, economic and human burden. In western countries, injury rates are higher among HCWs than among workers in any other field. ${ }^{56}$ Some classic occupational hazards seem to re-emerge owing to societal changes, including biological hazards (such as hepatitis B virus or HBV, hepatitis C virus or HCV, human immunodeficiency virus or 
HIV, measles, influenza, varicella and tuberculosis infections, among others). ${ }^{7-15}$ These constitute a major occupational health problem, as most of them are bloodborne diseases which can be acquired through needle-stick or sharps injuries. HCWs have contact with infected patients and their body fluids. Moreover, they frequently perform exposure-prone procedures that may cause injury. This risk involves surgeons, midwives, microbiologists, pathologists and blood bank and dialysis staff, among others. According to a recent review by Cooke and Stephens, in 2015 a needle-stick injury generated a cost of $\$ 747$ (range \$199-\$1691). ${ }^{16}$ Prüss-Ustün and collaborators used mathematical modelling to estimate the global burden of infections due to percutaneous injuries (PIs) among HCWs. Their model was based on the probability of injury, the prevalence of infection, the susceptibility of the worker and the potential of percutaneous transmission. In western countries, 1510 cases of HCV, 360 cases of HBV and 11 cases of HIV occurred in the year 2000 , accounting for $8 \%-27 \%, 1 \%-8 \%$ and $0.5 \%-3.1 \%$ of infections, respectively. ${ }^{17} \mathrm{~A}$ recently published systematic review and meta-analysis of the literature performed by Auta and coworkers ${ }^{18}$ computed a global 1-year prevalence of PIs of $36.4 \%$ (95\% CI 32.9 to 40.0), ranging from 9.5\% (95\% CI 6.7 to 12.4 ) in Australasia to $15.7 \%$ (95\% CI 12.1 to 19.3) in North America and to $31.8 \%$ (95\% CI 25.0 to 38.5) in Europe. Incidence rates of sharps injuries ranged from 1.4 to 9.5 per $100 \mathrm{HCWs}$, with a weighted mean of 3.7/100 HCWs per year and a related mean societal cost of $€ 272 .{ }^{19}$ According to Deuffic-Burban and colleagues, ${ }^{20}$ the risk of transmission of bloodborne pathogen infections in susceptible HCWs (ie, without postexposure prophylaxis or adequate hepatitis $\mathrm{B}$ vaccination) is estimated to be $30 \%, 0 \%-0.5 \%$ and $<0.3 \%$ for HBV, HCV and HIV, respectively. Other biological risks, such as airborne pathogen infections, pose serious health risks for both HCWs and patients: in recent years, large measles outbreaks, with nosocomial transmission among HCWs, have been documented in Italy and the UK. ${ }^{21} 22$

The incidence of occupational exposure, and, therefore, of PIs can be reduced by adopting preventive measures, such as taking standard precautions or implementing training sessions targeting both long-term HCWs and students and residents at risk. For instance, in France, the proportion of PIs preventable by means of standard precautions decreased from $52.5 \%$ in 2004 to $45.8 \%$ in $2008 .^{20}$

Other hazards are ergonomic/physical. Musculoskeletal injuries caused by patient handling and overexertion are among the most frequent traumas sustained by HCWs. Musculoskeletal injuries, which may involve muscles, nerves, tendons and ligaments, joints and cartilage, are due to such factors as repetitive movements, force, awkward postures, contact stress or vibration. Personnel assigned to hospital housekeeping, laundry and food services, maintenance, central supply and office tasks, as well as those involved in patient care, are susceptible to such injuries. Patient-handling injuries may be caused by manually lifting patients, who are generally more overweight or obese than in the past. Consequently, a 'Safe Patient Handling-No Manual Lifting' policy should be adopted. This is especially important today, given the current shortage of HCWs, particularly of nurses, and the need to mobilise patients early and to assist them with physical activities. ${ }^{23}{ }^{24}$ According to some epidemiological surveys, up to two-thirds of nurses have suffered from musculoskeletal disorders for at least 14 days at least once in their working lives. ${ }^{25}$ Physiotherapists are also at high risk of developing musculoskeletal disorders: according to a recent comprehensive narrative review, their lifetime and yearly prevalence rates are 55\%-91\% and $40 \%-91.3 \%$, respectively. Injuries generally affect the lower back, neck, upper back and shoulders. ${ }^{26}$

Physical violence is another occupational hazard, and severely impacts on HCWs' well-being and job motivation, affecting healthcare provision and quality. ${ }^{27}$ According to WHO estimates, from $8 \%$ to $38 \%$ of HCWs have been assaulted by patients or visitors at least once in their careers. Recently, the WHO, the 'International Labour Organization', the 'International Council of Nurses' and the 'Public Services International' jointly drafted a document entitled 'Framework guidelines for addressing workplace violence in the health sector'. ${ }^{28}$ The risk of physical violence is particularly high among HCWs working in psychiatric wards. ${ }^{29}$ Indeed, according to a recent review of the literature, lifetime rates of overall assaults, physical and verbal threats and sexual harassment in acute psychiatric units are $24 \%-80 \%$, $46 \%-78.6 \%, 43 \%-78.6 \%$ and $9.5 \%-37.2 \%$, respectively. The complications of such episodes include fractures, eye injuries and permanent disability, as well as psychological symptoms such as anxiety, depression, post-traumatic stress disorder or avoidance behaviour. ${ }^{30} 31$ Another environment in which the risk of violence/assault is high is the emergency department (ED): according to a recent qualitative meta-synthesis, ED staff members perceive aggression as unavoidable and feel that they are too often left to handle it alone. ${ }^{32}$ Aggressors typically suffer from psychiatric disorders, have a history of drug or alcohol use, carry weapons, are themselves victims of violence and are unable to cope rationally with situational crises. ${ }^{33} \mathrm{In}$ recent years, the number of assaults against HCWs has risen. For example, in one university teaching hospital in northern Italy, non-fatal violent events increased from 20.65/10 000 in 2012 to 22.81/10 000 in 2014, resulting in 431 days of absence from work and generating a direct cost of $€ 64170$. Up to $75 \%$ of violent episodes occurred in the ED, intermediate care, psychiatry and geriatrics wards. $^{34}$

A less common source of injury among HCWs is exposure to chemicals (inhalation of anaesthetics, solvents, detergents or reagents) ${ }^{35}$ or physical agents (such as ionising and non-ionising radiations). ${ }^{36}$

A particular type of disorder is also known to affect shift workers. Research among shift and non-shift workers has revealed a strict, statistically significant relationship 
between shift work and excess daytime sleepiness. ${ }^{37-40}$ Owing to workforce shortages, high workloads and the need to work at night, $32 \%$ of HCWs report not getting enough sleep. ${ }^{40}$

Systematically identifying working conditions associated with exposure to health hazards and subsequently taking remedial actions can play a major role in primary prevention. $^{41}$

\section{Review objectives}

The objectives of our planned study will be to: (1) provide a comprehensive overview of all studies dealing with injuries among HCWs in western countries; (2) identify the most frequent kinds of injuries among HCWs; (3) identify the type(s) of HCWs most prone to injuries; (4) identify which variable(s) impact(s) on the occurrence of injuries among HCWs; (5) quantify the burden of injuries among HCWs in terms of related disabilities, residual working capability, absence from work and direct/indirect costs generated; (6) identify preventive measures that can effectively curb the occurrence of injuries among HCWs; and (7) disseminate review findings in the published literature on injuries among HCWs.

\section{Review questions}

The review questions of our planned study are: (1) What is the incidence/prevalence rate of injuries among HCWs in western countries? (2) What are the determinants of injuries among HCWs in western countries? (3) What type of injury most commonly occurs among HCWs in western countries? (4) Among the different professional figures within the umbrella term of HCWs, which one(s) is/are the most affected by injuries in western countries? (5) What is the burden imposed by injuries among HCWs in terms of related disabilities, residual working capability, absence from work and direct/indirect costs generated? (6) What are the state-of-art preventive measures that can be adopted in order to effectively reduce injuries among HCWs in western countries?

\section{METHODS}

\section{Study design}

In order to properly address the research questions, a scoping review will be performed by means of the six-stage methodological framework initially proposed by Arksey and O'Malley. ${ }^{42}$ This framework comprises six steps: namely, (1) identifying the research question(s); (2) identifying relevant studies; (3) study selection; (4) charting the data; (5) collating, summarising and reporting the results; and (6) consultation exercise. ${ }^{43} 44$ This conceptual scheme has been made more detailed and explicit by Levac et al, ${ }^{45}$ further refined by Colquhoun $e t a l,{ }^{46}$ and subsequently modified by the Joanna Briggs Institute (JBI) in the 'JBI scoping review methods' manual. ${ }^{46} 47$

The specific methodology of the scoping review ${ }^{47} 48$ was chosen in order to take into account both the nature and the specific requests of a national project co-funded and performed in collaboration between the Department of Health Sciences, Occupational Medicine-University of Genoa, Italy, and the National Institute for Insurance Against Accidents at Work (in Italian, 'Istituto Nazionale per l'Assicurazione contro gli Infortuni sul Lavoro', INAIL). This objective requires the rapid assessment of a large amount of diverse scholarly literature, the aim being to achieve breadth rather than depth. For this reason, other types of review, such as systematic reviews, umbrella reviews or rapid reviews, ${ }^{49}$ were not deemed methodologically effective.

As maintained by Arksey and O'Malley, ${ }^{42}$ scoping reviews may be used to assess the topology of a vast body of literature, in order to identify current gaps of knowledge and future prospects. ${ }^{4250}$ These aims corresponded to the objectives of our project.

\section{Drafting and registration of the study protocol}

This protocol is reported in accordance with the Preferred Reporting Items for Systematic Reviews and Meta-Analysis-Protocols (PRISMA-P) guidelines. $^{51}$ However, despite the recommendation of these guidelines, it was not possible to register the scoping review protocol in the International Prospective Register of Systematic Reviews, ${ }^{52}$ in that this register does not currently accept scoping review protocols.

\section{Stage I: Identifying the research question(s)}

Five authors (NLB, GD, VM, AT and PD) drafted the research questions; the other authors (VP, CB, RL, FB, $\mathrm{BDA}, \mathrm{EM}, \mathrm{AM}$ and $\mathrm{ND}$ ) helped to develop these questions. The research objectives and questions have been formulated as they are stated in the previous sections 'Review objectives' and 'Review questions'.

\section{Stage II: Identifying relevant studies}

The identification of relevant studies will follow the three-step process recommended by the JBI: namely, (1) preliminary search conducted on at least two databases; (2) preparation of a list of search terms and words to be used in the subsequent running of the search on a larger number of databases; and (3) possible additional searches (cross-checking/cross-referencing of reference lists of potentially eligible studies, hand-searching in target journals relevant to the topic, etc).

\section{Preliminary literature search}

We carried out a preliminary literature search on the topic of interest in order to clarify inclusion/exclusion criteria. After familiarising ourselves with the literature, we were able to further expand and/or modify/refine the search strategy with the help of an expert, qualified research librarian.

The preliminary literature search was carried out on two widely used scholarly databases (PubMed/MEDLINE and Scopus), using 'healthcare injuries' as keywords and adopting a time filter; this yielded 27844 and 139073 studies, respectively. In the second stage, the research team inspected titles and abstracts of potentially relevant 
articles and prepared a list of pertinent words and index terms to inform the subsequent search process.

\section{Structured search strategy}

Based on the previously prepared list of key terms, a systematic literature search will be carried out on several scholarly databases, namely PubMed/MEDLINE (NLM), Scopus, SciVerse ScienceDirect, Science Citation Index Expanded and Social Sciences Citation Index from the ISI/Web of Science, ProQuest Research Library, ABI/ INFORM, CBCA, via the UNO per TUTTI Primo Central (Ex Libris) platform databases.

We will include all studies reporting epidemiological figures of injuries among HCWs in western countries in terms of prevalence/incidence rates. The search will be performed by using an appropriate string of relevant search terms based on controlled vocabulary and Boolean connectors (table 1). For PubMed/MEDLINE, for instance, Medical Subject Headings key terms and wild card option (truncated key terms) will be used. This structured search strategy will be adapted to the other databases, in order to obtain database-specific search strategies. If a decision on the inclusion or exclusion of a study cannot be made on the basis of the abstract, the full text of the article will be examined. During this process, we will use the Peer Review of Electronic Search Strategies 2015 Guideline statement as a guide. ${ }^{53}$

In the third phase of the search strategy, we will scan reference lists of the studies chosen and prior reviews. Additionally, we will hand-search target journals relevant to the topic under study.

Five authors (NLB, GD, VM, AT and PD) will identify relevant studies, with the aid of the other authors (VP, CB, RL, FB, BDA, EM, AM and ND).

\section{Stage III: Study selection}

Once the search strategy has been successfully completed, search results will be collated and exported to EndNote V.X7 (Clarivate Analytics). Duplicates will be automatically removed before the file containing a set of unique records is made available to reviewers for further processing (ie, study screening and selection).

The studies will be independently screened by two authors (NLB and GD), who will read study titles and abstracts for potential eligibility. Screening questions will be drafted and pilot-tested on a subset of records randomly chosen before implementation. Inter-rater agreement will be assessed by means of the $\kappa$ statistic and any disagreement will be resolved through discussion; a third reviewer $(\mathrm{PD})$, acting as a final referee, will be involved if necessary.

\section{Inclusion criteria}

Studies meeting the following PECO criteria will be considered for inclusion ${ }^{54}$ :

- P (patient, problem or population): HCWs (of any type, medical, nursing or dental practitioners, trainees/residents, other allied health professionals, central supply workers and technicians) working in western countries.

- E (exposure): injuries (of any type, due to exposure to biological, chemical, physical/ergonomic and any other potential risk and hazard).

- C (comparison, control or comparator): different types of HCWs (medical versus nursing or dental practitioners, trainees or residents); exposed HCWs versus non-exposed; before and after a preventive programme.

- O (outcome/outcomes of interest): prevalence/incidence and determinants of injuries, occupational burden (in terms of related disabilities and absence from work) and economic burden generated (in terms of direct and indirect costs).

Furthermore, the following criteria will be taken into consideration:

- Study design/characteristics: original articles, prevalence/incidence studies.

- Time: a time filter/restraint will be applied. Only papers written between 2000 and 2018 will be considered.

- Languages: no language filter/restraint will be applied. Non-English articles that are included will be acquired in full text and translated by expert translators from the University of Genoa, Italy.

\section{Exclusion criteria}

Articles that do not meet the above-stated PECO criteria, or which provide insufficient information, and studies designed as editorials, letters to the editor, commentaries, expert opinions, case reports, case series and reviews will be excluded.

\section{Reporting the studies selected}

Details of the literature search and screening results will be both summarised narratively and presented graphically by means of PRISMA flow diagram (figure 1) ${ }^{55}$ In addition, we will provide a table of excluded studies, with reasons for their exclusion, in our published final scoping review.

\section{Stage IV: Charting the data}

An ad hoc data-extraction template reflecting the research questions and the purposes/objectives of the review will be created. This will be used both to confirm study relevance and to extract the data. In detail, the template will gather information regarding the key characteristics of the studies, such as study authors, year of publication, study population (type(s) of HCWs recruited) and study country, study design, percentage of male HCWs, mean age, sample size, attrition rate, years of professional experience, working setting, prevalence/incidence rate of injuries, knowledge, attitudes and practices concerning the adoption of standard procedures, and injury-related burden (table 2). These a priori data items have been individuated and developed through a preliminary exercise by all the research team. 
Table 1 Planned search strategy

\section{Search strategy item Search strategy}

\begin{tabular}{|c|c|}
\hline Databases & $\begin{array}{l}\text { PubMed/MEDLINE (NLM), Scopus, SciVerse ScienceDirect, Science Citation Index Expanded and } \\
\text { Social Sciences Citation Index from ISI/Web of Science, ProQuest Research Library, ABI/INFORM, } \\
\text { CBCA, via the UNO per TUTTI Primo Central (Ex Libris) platform }\end{array}$ \\
\hline Language filter & None \\
\hline Time filter & $2000-2018$ \\
\hline Spatial filter & Western countries \\
\hline Keywords & $\begin{array}{l}\text { 1. "healthcare worker" OR "healthcare workers" OR "healthcare personnel" OR "healthcare staff" } \\
\text { OR "health worker" OR "health workers" OR "health personnel" OR "health staff" OR physicians OR } \\
\text { physician OR doctors OR doctor OR nurses OR nurse OR practitioners OR practitioner OR "medical } \\
\text { students" OR "medical residents" OR "attending residents" OR "hospital technician" OR "hospital } \\
\text { technicians" OR "paramedical personnel" OR "paramedical staff" OR "hospital support personnel" } \\
\text { 2. injury OR injuries OR incident OR incidents OR "occupational injury" OR "occupational injuries" } \\
\text { OR "occupational incident" OR "occupational incidents" OR "work related injury" OR "work related } \\
\text { injuries" OR "work related incident" OR "work related incidents" OR "workplace-induced injury" OR } \\
\text { "workplace-induced injuries" OR "workplace-induced incident" OR "workplace-induced incidents" OR } \\
\text { "occupational health hazard" OR "occupational health hazards" } \\
\text { 3. "exposure incidents" OR "splash exposures" OR "splash exposure" OR "needle-sticks" OR "sharp } \\
\text { objects" OR "sharps" OR "percutaneous injuries" OR "percutaneous injury" } \\
\text { 4. "manual handling injury" OR "manual handling injuries" OR "musculoskeletal injury" OR } \\
\text { "musculoskeletal injuries" } \\
\text { 5. "chemical occupational exposure" OR "exposure to inhaled anesthetic" OR "reagent exposure" OR } \\
\text { "exposure to reagent" OR "exposure to solvents" OR "solvent exposure" OR "exposure to detergents" } \\
\text { OR "detergent exposure" } \\
\text { 6. "slips, trips and falls" OR "slipping, tripping and falling accidents" OR "accidental fall" OR "same- } \\
\text { level fall" OR "same-surface fall" OR "stump-and-fall" OR "step-and-fall" OR "forced-rotation-type fall" } \\
\text { OR "fall from elevation" } \\
\text { 7. "violent events" OR "violence" OR "assault" OR "assaults" } \\
\text { 8. "cuts and wounds" OR "burns" } \\
\text { 9. "motor vehicle accidents" OR "motor vehicle accident" OR "motor vehicle collisions" OR "motor } \\
\text { vehicle collision" OR "motor vehicle crash" OR "motor vehicle crashes" OR "motor vehicle near crash" } \\
\text { OR "motor vehicle near crashes" } \\
\text { 10. "exposure to ionizing radiation" OR "radiation exposure" } \\
\text { 1. AND 2. OR 3. OR 4. OR 5. OR 6. OR 7. OR 8. OR 9. OR 10. }\end{array}$ \\
\hline Inclusion criteria & $\begin{array}{l}\text { P: Medical/dental/paramedical students and residents, doctors, nurses and other allied health } \\
\text { professionals, cleaners and porters, central supply workers and technicians. } \\
\text { E: Exposure to biological, chemical, physical/ergonomic and any other potential risk and hazard. } \\
\text { C: Medical versus nursing or dental students; students versus residents; medical versus nursing } \\
\text { or dental trainees/residents; exposed HCWs versus non-exposed; before and after a preventive } \\
\text { programme. } \\
\text { O: Prevalence/incidence of injuries and their determinants among healthcare workers in western } \\
\text { countries, related disabilities and absence from work, and economic burden generated (direct/indirect } \\
\text { costs). } \\
\text { Study design: Primary research. }\end{array}$ \\
\hline Exclusion criteria & $\begin{array}{l}\text { Studies not meeting the above-stated patient/exposure/comparator/outcome (PECO) criteria. } \\
\text { Study design: Editorial, letter to the editor, commentary, case report, case series, review. }\end{array}$ \\
\hline & \\
\hline
\end{tabular}

HCW, healthcare worker.

The data-extraction process will be performed independently by two authors (NLB and GD) and will be pilot-tested on a small sample of randomly selected studies, until consensus is reached. On the basis of this pilot test, if deemed necessary by the research team, the data-extraction form will be reviewed and revised, in order to capture any relevant information contained in the studies included. Any change to the data-collection form will be documented and explained. To assist this process of the scoping review, the 'Covidence' software (Cochrane) for systematic review management will be used.

Stage V: Collating, summarising and reporting the results

Stage $\mathrm{V}$ is aimed at providing a summary and synthesis of the findings. However, Levac et $a l^{45}$ have suggested 


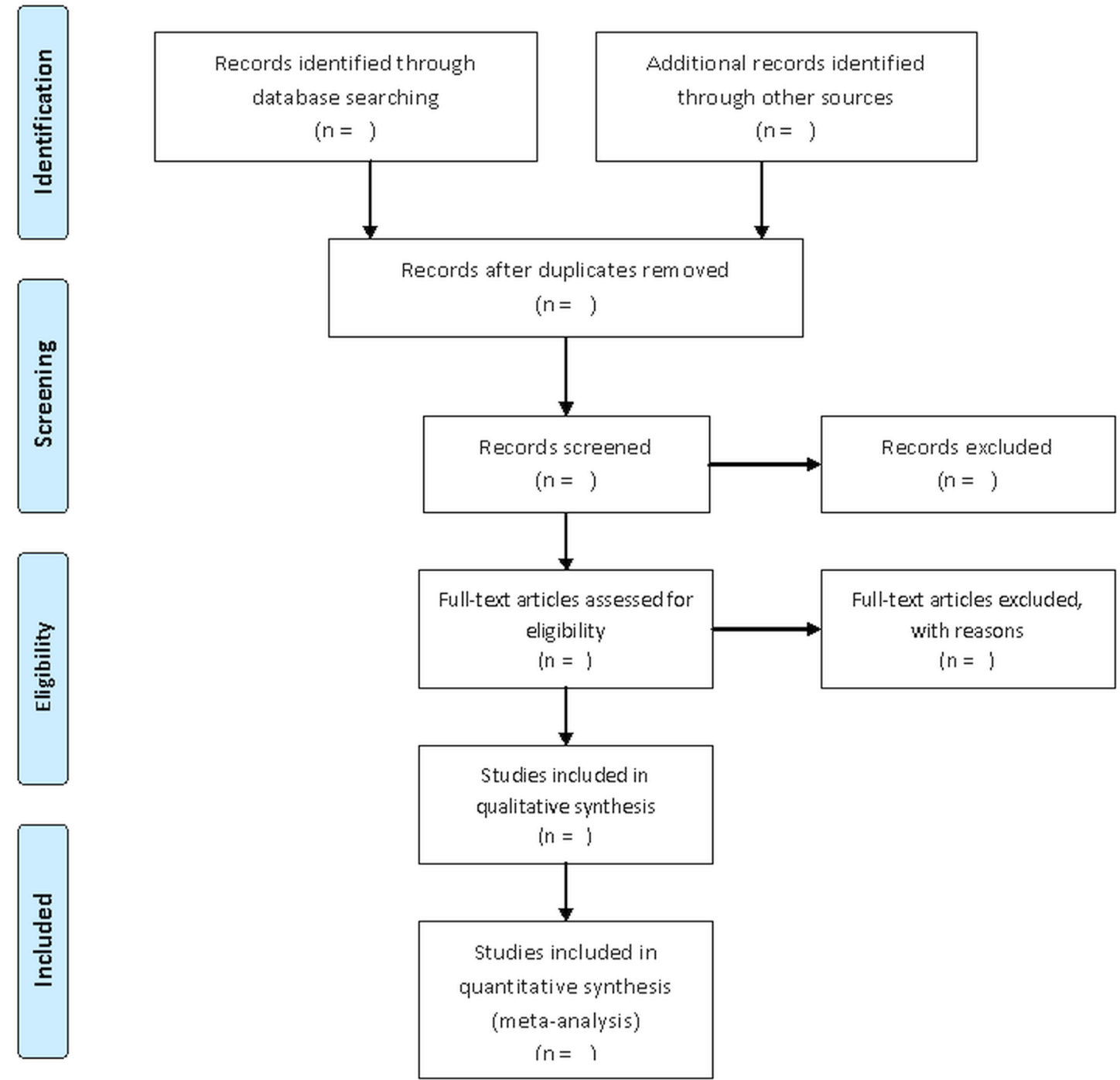

Figure 1 Flow chart of study selection in accordance with the Preferred Reporting Items for Systematic Reviews and MetaAnalyses guidelines.

breaking down this review step into the following three smaller and distinct phases: namely, (a) data collation and analysis; (b) reporting of the results and outcome(s) in such a way as to guide and inform the overall study purposes/objectives, and research questions; and (c) taking into account the meaning of the findings in relation to the study purposes/objectives and research questions, and discussion of the potential, practical implications that the findings may have on future research, practice and policy.

The data collected during stage IV will be stored in an Excel electronic database. In addition to the narrative review describing how the results relate to the review purposes/objectives and questions, we will provide a table showing the main characteristics of the studies included in the scoping review. We will also assess the frequency of studies investigating: (1) the type(s) of HCWs recruited, (2) the types of injuries and (3) the outcomes studied (injury rate and determinants; burden generated by the injuries; and preventive measures that can be adopted to curb the injury rate).
Findings will be reported according to the PRISMA Extension for Scoping Reviews checklist. ${ }^{56}$

\section{Stage VI: Consultation exercise and stakeholder involvement}

Both Arksey and O'Malley ${ }^{42}$ and Levac et $a t^{45}$ have suggested that the consultation exercise stage can provide opportunities to involve key stakeholders, providing insights beyond those that can be found in the scholarly literature. The development of our planned scoping review will include the ongoing involvement of relevant scientific societies, such as the Italian Society of Occupational Medicine ('Società Italiana di Medicina del Lavoro'), and of the national and international networks of occupational physicians. Prior to publication and dissemination of the findings, we will once again consult with these stakeholders in order to receive their feedback and to ensure that the data have been clearly and accurately presented.

\section{Patient and public involvement}

In the development of the planned scoping review, there will be no specific involvement of patients or the public. 
Table 2 Data to be extracted and details/explanations

\begin{tabular}{|c|c|}
\hline Extracted data & Details \\
\hline Study reference & Names and surnames of authors, year of publication. \\
\hline Study population & $\begin{array}{l}\text { Physicians, doctors, nurses, medical, dental students, residents, other allied health } \\
\text { professionals, cleaners, porters, central supply workers and technicians. }\end{array}$ \\
\hline Country & Western country or countries in which the study or studies was or were carried out. \\
\hline Study design & Type of recruitment. \\
\hline $\mathrm{M} \%$ & Percentage of male healthcare workers. \\
\hline Age & Mean age of healthcare worker sample. \\
\hline Sample number, attrition rate & Number of healthcare workers who took part in the survey, number of non-responders. \\
\hline Professional/experience years & Years spent in profession by healthcare workers included in the study. \\
\hline Working setting & $\begin{array}{l}\text { Hospital ward where the injury occurred (eg, emergency room, obstetrics department, } \\
\text { surgery department, operating room, outpatient clinic, department of internal medicine, } \\
\text { patient's room, CCU/ICU). }\end{array}$ \\
\hline Injury prevalence/incidence rate & Prevalence/incidence rates stratified according to the kind of injury. \\
\hline Method & Questionnaire (validated, not validated). \\
\hline $\begin{array}{l}\text { Compliance with standard } \\
\text { procedures }\end{array}$ & $\begin{array}{l}\text { Prevalence/incidence rates stratified according to the compliance with procedures and } \\
\text { guidelines among the different types of healthcare workers. }\end{array}$ \\
\hline $\begin{array}{l}\text { Knowledge, attitudes and } \\
\text { practices regarding PEP }\end{array}$ & $\begin{array}{l}\text { Prevalence/incidence rates stratified according to knowledge, attitudes and practices } \\
\text { concerning PEP, among the different types of healthcare workers. }\end{array}$ \\
\hline Clerkship abroad & $\begin{array}{l}\text { Periods of training abroad; type of task(s) the healthcare worker was involved in during the } \\
\text { training period abroad. }\end{array}$ \\
\hline $\begin{array}{l}\text { Reporting/non-reporting to } \\
\text { occupational department }\end{array}$ & $\begin{array}{l}\text { Prevalence/incidence rates stratified according to the determinants of reporting/non- } \\
\text { reporting, among the different types of healthcare workers. }\end{array}$ \\
\hline Injury-related burden & $\begin{array}{l}\text { Number of days of absence from work, disabilities and direct/indirect economic costs due } \\
\text { to injuries. }\end{array}$ \\
\hline
\end{tabular}

CCU, critical care unit; ICU, intensive care unit; PEP, postexposure prophylaxis.

\section{DISCUSSION}

\section{Implications}

HCWs are subject to various risk factors and risky behaviours, which may have a serious impact on their health and safety. Moreover, both non-fatal and fatal work-related injuries and illnesses impose an economic burden on society. ${ }^{56-58}$ Thus, a major challenge for western countries is to promote the health and well-being of individuals at both the occupational and community levels, in order to enable workers to stay at work longer and in good health. ${ }^{5960}$ Mapping the existing literature on injuries in the healthcare setting enables us to understand both traditional and emerging health problems at work (such as the impact of ageing, musculoskeletal and psychosocial problems, shift work, gender perspectives, re-emergent infectious diseases) and provides useful insights into their determinants. This approach underpins the planning and implementation of highquality occupational health interventions in the currently changing world of work. ${ }^{61}$

The proposed scoping review is expected to contribute to the existing scholarly literature through its potential to inform and influence healthcare practice, education and policy and to guide future research in the field.
As yet, no reviews of studies conducted in western countries concerning injuries among HCWs have been carried out. As such, this scoping review will provide the first rigorous analytical, updated synthesis of primary research data on the epidemiology and the economic and occupational burden of injuries among HCWs in western countries. Stratifying injuries according to type and the work tasks involved could add meaningful information and increase our understanding of the determinants of injuries among HCWs.

Owing to the changing conditions of our society and of healthcare settings, new hazards are emerging alongside classic occupational risk factors and ensuring health protection in the workplace is mandatory. Acquiring good evidence concerning the epidemiology of injuries and associated determinants among HCWs in western countries is the scientific basis for implementing programmes and properly orienting the activities of occupational health services and policies. This would bring considerable benefits not only to workers but to entire organisations and societies, which is an objective of occupational and public health programmes.

Furthermore, this scoping review will allow us to assess the scholarly literature for knowledge gaps that researchers will be able to address in their future 
research. Being conducted in partnership with the INAIL and the national and international networks of occupational physicians, the planned scoping review will also be useful to decision- and policy-makers, in order to design, develop, implement and foster adequate, cost-effective ad hoc policies and practices for primary prevention and educational programmes.

\section{Strengths and limitations}

The main strength of this scoping review lies in the rigour, transparency and reproducibility of our approach. Indeed, it will be based on the present scoping review protocol, which has been submitted separately for review and publication, in order to ensure high methodological standards. Any amendments to the present scoping review protocol will be precisely documented, listed and explained in the final review publication(s).

Another strength is the multidisciplinary nature of our team, which comprises an experienced epidemiologist and research methodologist (NLB), a biologist with a background in the field of public health (VP), occupational physicians from the academic setting (GD, AT, FB, BDA, EM, AM, ND and PD) and occupational physicians with expertise in the field of social security and insurance (CB, RL and VM).

However, some shortcomings must also be mentioned. The main limitation concerns the time filter (studies published between 2000 and 2018 will be included in the scoping review). However, this will enable us to make an updated, direct comparison of the findings with the epidemiological figures available at national and local INAIL centres. Moreover, as the process of scoping reviews does not include formal critical quality assessment and appraisal of the studies included, the findings reported may lack reliability and validity.

\section{DISSEMINATION}

Following the successful completion of the scoping review, its findings will be submitted to peer-reviewed journals for potential publication(s) and will be the subject of $a d$ hoc oral/poster communication(s) in relevant national and international scientific congresses and conferences. All members of the research team have established relationships with national and international occupational medicine networks which will also be used to further disseminate the review findings.

We will not be able to provide any recommendations, since the studies selected will not be critically and formally appraised for methodological quality. The findings of this scoping review could be used to guide the education of HCWs (eg, to inform the development and implementation of courses for continuous medical learning) and the health policy-making and decision-making process.

\footnotetext{
Author affiliations

${ }^{1}$ Department of Health Sciences (DISSAL), Postgraduate School of Occupational

Medicine, University of Genoa, Genoa, Italy

${ }^{2}$ Occupational Medicine Unit, Policlinico San Martino Hospital IRCCS, Genoa, Italy
}

${ }^{3}$ Liguria Regional Directorate, National Institute for Insurance Against Accidents at Work/ Istituto nazionale per l'assicurazione contro gli infortuni sul lavoro (INAIL), Genoa, Italy

${ }^{4}$ Occupational Medical Service, Local Health Unit 1, Liguria Regional Healthcare System, Imperia, Italy

Contributors NLB, GD, VP, CB, RL, VM, AT, FMB, BD'A, EM, AM, ND and PD conceived the idea. NLB, GD, VM, AT and PD developed the research questions. NLB, GD and PD developed the study methods. VP, CB, RL, FB, BDA, EM, AM and $\mathrm{ND}$ aided in developing the research question and study methods. All authors participated in drafting the article, editing or revising it critically for important intellectual content; all authors gave final approval of the version to be submitted and any revised version.

Funding This research was co-funded by the "Istituto Nazionale per I'Assicurazione contro gli Infortuni sul Lavoro (INAIL, National Institute for Insurance Against Accidents at Work)" and the Department of Health Sciences, Occupational Medicine - University of Genoa, Italy.

Competing interests None declared.

Patient consent Not required.

Ethics approval No ethical clearance is required for the present scoping review protocol and for its subsequent implementation, in that it will summarise knowledge and analyse data that have been already collected and published.

Provenance and peer review Not commissioned; externally peer reviewed.

Open access This is an open access article distributed in accordance with the Creative Commons Attribution Non Commercial (CC BY-NC 4.0) license, which permits others to distribute, remix, adapt, build upon this work non-commercially, and license their derivative works on different terms, provided the original work is properly cited, appropriate credit is given, any changes made indicated, and the use is non-commercial. See: http://creativecommons.org/licenses/by-nc/4.0/.

\section{REFERENCES}

1. World Health Organization. Working Together for Health: World Health Report. Geneva: Switzerland, 2006.

2. Joseph B, Joseph M. The health of the healthcare workers. Indian J Occup Environ Med 2016;20:71-2.

3. Adams BO, Dal Poz MR, Shengelia B, et al. Human, Physical, and Intellectual Resource Generation: Proposals for Monitoring. In: Murray CJL, Evans D, eds. Health Systems Performance Assessment: Debates, Methods and Empiricism. Geneva: World Health Organization, 2003:273-87.

4. Diallo K, Zurn P, Gupta N, et al. Monitoring and evaluation of human resources for health: an international perspective. Hum Resour Health 2003;1:3.

5. Dressner MA. Hospital workers: an assessment of occupational injuries and illnesses. Monthly Labor Review, U.S. Bureau of Labor Statistics, 2017.

6. Miller K. Risk factors and impacts of occupational injury in healthcare workers: A critical review. OA Musculoskeletal Medicine 2013;1:4.

7. Young TN, Arens FJ, Kennedy GE, et al. Antiretroviral post-exposure prophylaxis (PEP) for occupational HIV exposure. Cochrane Database Syst Rev 2007;1:CD002835.

8. Rischitelli G, Harris J, McCauley L, et al. The risk of acquiring hepatitis $\mathrm{B}$ or $\mathrm{C}$ among public safety workers: a systematic review. Am J Prev Med 2001;20:299-306.

9. Westermann C, Peters C, Lisiak B, et al. The prevalence of hepatitis $\mathrm{C}$ among healthcare workers: a systematic review and meta-analysis. Occup Environ Med 2015;72:880-8.

10. Dini G, Toletone A, Sticchi L, et al. Influenza vaccination in healthcare workers: A comprehensive critical appraisal of the literature. Hum Vaccin Immunother 2017;8:1-18.

11. Uden L, Barber E, Ford N, et al. Risk of tuberculosis infection and disease for health care workers: An updated meta-analysis. Open Forum Infect Dis 2017;4.

12. Placidi D, Tonozzi B, Alessio L, et al. [Tuberculin skin test (TST) survey among healthcare workers (HCWs) in hospital: a systematic review of the literature]. G Ital Med Lav Ergon 2007;29(3 Suppl):409-11.

13. Riccò M, Vezzosi L, Odone A, et al. Invasive meningococcal disease on the workplaces: A systematic review. Acta Biomed 2017;88:337-51.

14. van den Hoogen A, Duijn JM, Bode LGM, et al. Systematic review found that there was moderate evidence that vaccinating healthcare 
workers prevented pertussis in infants. Acta Paediatr. In Press. 2018;107.

15. Leone Roberti Maggiore U, Scala C, Toletone A, et al. Susceptibility to vaccine-preventable diseases and vaccination adherence among healthcare workers in Italy: A cross-sectional survey at a regional acute-care university hospital and a systematic review. Hum Vaccin Immunother 2017;13:470-6.

16. Cooke CE, Stephens JM. Clinical, economic, and humanistic burden of needlestick injuries in healthcare workers. Med Devices 2017:10:225-35.

17. Prüss-Ustün A, Rapiti E, Hutin Y. Estimation of the global burden of disease attributable to contaminated sharps injuries among healthcare workers. Am J Ind Med 2005;48:482-90.

18. Auta A, Adewuyi EO, Tor-Anyiin A, et al. Global prevalence of percutaneous injuries among healthcare workers: a systematic review and meta-analysis. Int J Epidemiol. In Press. 2018;47:1972-80.

19. Elseviers MM, Arias-Guillén M, Gorke A, et al. Sharps injuries amongst healthcare workers: review of incidence, transmissions and costs. J Ren Care 2014;40:150-6.

20. Deuffic-Burban S, Delarocque-Astagneau E, Abiteboul D, et al. Blood-borne viruses in health care workers: prevention and management. J Clin Virol 2011;52:4-10.

21. Baxi R, Mytton OT, Abid M, et al. Outbreak report: nosocomial transmission of measles through an unvaccinated healthcare workerimplications for public health. J Public Health 2014;36:375-81.

22. Amendola A, Bianchi S, Frati ER, et al. Ongoing large measles outbreak with nosocomial transmission in Milan, northern Italy, March-August 2017. Euro Surveill 2017;22.

23. Edlich RF, Hudson MA, Buschbacher RM, et al. Devastating injuries in healthcare workers: description of the crisis and legislative solution to the epidemic of back injury from patient lifting. J Long Term Eff Med Implants 2005;15:225-42.

24. Nelson AL, Collins J, Knibbe H, et al. Safer patient handling. Nursing Management 2007;38:26-32.

25. Delloiacono N. Musculoskeletal safety for older adults in the workplace: review of current best practice evidence. Workplace Health Saf 2015;63:48-53.

26. Milhem M, Kalichman L, Ezra D, et al. Work-related musculoskeletal disorders among physical therapists: A comprehensive narrative review. Int J Occup Med Environ Health 2016;29:735-47.

27. Dillon BL. Workplace violence: impact, causes, and prevention. Work 2012;42:15-20.

28. International Labour Office (ILO), International Council of Nurses (ICN), World Health Organization (WHO), Public Services International (PSI). Joint Programme on Workplace Violence in the Health Sector. Geneva, 2002.

29. Spaducci G, Stubbs B, McNeill A, et al. Violence in mental health settings: A systematic review. Int J Ment Health Nurs 2018;27:33-45.

30. d'Ettorre G, Pellicani V. Workplace violence toward mental healthcare workers employed in psychiatric wards. Saf Health Work 2017;8:337-42

31. Luftman K, Aydelotte J, Rix K, et al. PTSD in those who care for the injured. Injury 2017;48:293-6.

32. Ashton RA, Morris L, Smith I. A qualitative meta-synthesis of emergency department staff experiences of violence and aggression. Int Emerg Nurs. In Press. 2018;39:13-19.

33. Gillespie GL, Gates DM, Miller M, et al. Workplace violence in healthcare settings: risk factors and protective strategies. Rehabil Nurs 2010;35:177-84.

34. Sossai D, Molina FS, Amore M, et al. Analysis of incidents of violence in a large Italian hospital. Med Lav 2017;108:6005.

35. Molina Aragonés JM, Ayora Ayora A, Barbara Ribalta A, et al. Occupational exposure to volatile anaesthetics: a systematic review. Occup Med 2016;66:202-7.

36. Caciari T, Capozzella A, Tomei F, et al. Professional exposure to ionizing radiations in health workers and white blood cells. Ann Ig 2012:24:465-74

37. Garbarino S, Traversa F, Spigno F, et al. [Sleepiness, sleep disorders and risk of occupational accidents]. G Ital Med Lav Ergon 2011(33(3 Suppl):207-11.
38. Garbarino S, Repice AM, Traversa F, et al. [Commuting accidents: the influence of excessive daytime sleepiness. A review of an Italian Police officers population]. G Ital Med Lav Ergon 2007;29(3 Suppl):324-6.

39. Caruso CC. Negative impacts of shiftwork and long work hours. Rehabil Nurs 2014:39:16-25.

40. Booker LA, Magee M, Rajaratnam SMW, et al. Individual vulnerability to insomnia, excessive sleepiness and shift work disorder amongst healthcare shift workers. A systematic review. Sleep Med Rev 2018;41:220-33.

41. Copello F, Garbarino S, Messineo A, et al. Occupational medicine and hygiene: applied research in Italy. J Prev Med Hyg 2015;56:E102-10.

42. Arksey H, O'Malley L. Scoping studies: towards a methodological framework. Int J Soc Res Methodol 2005;8:19-32.

43. Khalil H, Peters M, Godfrey CM, et al. An evidence-based approach to scoping reviews. Worldviews Evid Based Nurs 2016;13:118-23.

44. Levac D, Colquhoun H, O'Brien KK. Scoping studies: advancing the methodology. Implement Sci 2010;5:69.

45. Colquhoun HL, Levac D, O'Brien KK, et al. Scoping reviews: time for clarity in definition, methods, and reporting. $J$ Clin Epidemiol 2014;67:1291-4.

46. Tricco AC, Lillie E, Zarin W, et al. A scoping review on the conduct and reporting of scoping reviews. BMC Med Res Methodol 2016;16:15.

47. Peters MD, Godfrey CM, Khalil H, et al. Guidance for conducting systematic scoping reviews. Int J Evid Based Healthc 2015;13:141-6.

48. Grant MJ, Booth A. A typology of reviews: an analysis of 14 review types and associated methodologies. Health Info Libr J 2009;26:91-108.

49. Mays N, Roberts E, Popay J. et alSynthesising research evidence. In: Fulop N, Allen P, Clarke A, Black N, . eds. Studying the organisation and delivery of health services: Research methods. London: Routledge, 2001:188-219.

50. Peterson J, Pearce PF, Ferguson LA, et al. Understanding scoping reviews: Definition, purpose, and process. J Am Assoc Nurse Pract 2017;29:12-16.

51. Chien PF, Khan KS, Siassakos D. Registration of systematic reviews: PROSPERO. BJOG 2012;119:903-5.

52. McGowan J, Sampson M, Salzwedel DM, et al. PRESS Peer Review of Electronic Search Strategies: 2015 guideline statement. J Clin Epidemiol 2016;75:40-6.

53. Thabane L, Thomas T, Ye C, et al. Posing the research question: not so simple. Can J Anaesth 2009;56:71-9.

54. Moher D, Liberati A, Tetzlaff J, et al. Preferred reporting items for systematic reviews and meta-analyses: the PRISMA Statement. Open Med 2009;3:e123-30.

55. Tricco AC, Lillie E, Zarin W, et al. PRISMA Extension for Scoping Reviews (PRISMA-ScR): Checklist and Explanation. Ann Intern Med 2018;169:467-73.

56. European Agency for Safety and Health at Work (EU-OSHA). The economics of occupational safety and health - the value of OSH to society. 2017 https://visualisation. osha.europa.eu/osh-costs\#!/.

57. Heuvel S, Zwaan L, Lv D, et al. Estimating the costs of work-related accidents and ill-health: An analysis of European data sources. Luxembourg: European Agency for Safety and Health at Work (EUOSHA), 2017

58. Burton J. WHO Healthy workplace framework and model: Background and supporting literature and practices: World Health Organization, 2010.

59. Westerholm P, Walters D. Supporting Health at Work: International Perspectives on Occupational Health Services: IOSH Services, 2007.

60. Leijon O, Lindahl E, Torén $\mathrm{K}$, et al. First-time decisions regarding work injury annuity due to occupational disease: a gender perspective. Occup Environ Med 2014;71:147-53.

61. Steel J, Luyten J, Godderis L. Occupational Health: The Global Evidence and Value: Society of Occupational Medicine, International SOS Foundation, KU Leuven University, 2018. 